\title{
Forward
}

\section{There Are Still Plenty of Opportunities On the Boundaries}

This is the second of the two special issues of Electronic Journal of Boundary Elements in honor of Professor Frank Rizzo. There are thirteen technical papers in this issue, contributed by Professor Rizzo's colleagues, friends and former students. These papers cover a broad range of topics in the boundary integral equation and boundary element method (BIE/BEM), including Galerkin BEM for anisotropic elasticity (Gray, Griffith, et al.), evaluations of hypersingular integrals in Galerkin BEM (Bonnet and Guiggiani), Green's function BEM for bimaterials (Denda), new 3-D Green's functions for piezoelectric bimaterials (Pan), new formulations using local integral equations (Sladek and Sladek), BEM in sensitivity analysis with stress concentrations (Burczynski and Habarta), fracture of thermopiezoelectric materials (Qin), BEM for 3-D gradient elastodynamics (Polyzos, Tsepoura and Beskos), time-domain large-scale elastodynamic analysis (Yoshikawa and Nishimura), acoustic BEM for analyzing mufflers and silencers (Wu and Cheng), analysis of solids with randomly distributed inclusions (Yao, Kong and Zheng), thermal and stress analyses of thermal barrier coatings (Lu and Dong), and finally, modeling of carbon nanotube-based composites (Liu and Chen). These authors are gratefully acknowledged for their excellent contributions, and for their patience and cooperation in the process of preparing this special issue.

It is interesting to note that the wide applications of the elasticity BIE/BEM in engineering all started with a simple idea. That is, boundary-value problems can be solved by boundary-only methods. The first result in this direction is also amazingly concise. During a recent trip to Urbana, Illinois, I checked out Professor Rizzo's Ph.D. dissertation from the UIUC library. The thirty-page dissertation is without doubt a masterpiece that many current and future Ph.D. candidates may like to follow, for its originality and succinct writing. The dissertation laid a solid foundation for what is now called the BEM for elasticity and many other problems, and eventually led to the seminal paper of 1967. Behind this masterpiece are Professor Rizzo's affection and conviction in the BIE/BEM and his willingness to explore a different route in research. This spirit of exploration and his serious attitude in research have inspired and influenced many of his former students and colleagues in the last forty years.

Researchers in the pursuit of boundary-only methods can be described as explorers in a Flatland (see Professor Rizzo's article in Issue No.1). They can have different perspectives, but can also discover treasures that others could not uncover. As younger researchers come into this playground, new breakthroughs, just like the one made by Professor Rizzo in the 1960s, may not be far away. There will certainly be more innovative boundary-only methods emerging in the near future. More special numerical tools will be developed and more emerging problems will be solved by these new modeling tools. The fields of computational mechanics will be further diversified and thus prosperous. There are still plenty of opportunities on the boundaries!

Yijun Liu

University of Cincinnati 